\title{
Avaliação das infecções hospitalares em pacientes críticos em um Centro de Terapia Intensiva
}

\author{
Rui Barbosa Rodrigues CÂNDIDO ${ }^{1}$ \\ *Walnéia Aparecida de SOUZA ${ }^{2}$ \\ Márcia Helena Miranda Cardoso PODESTÁ ${ }^{2}$ \\ José Ricardo ROCHA \\ Vanessa Martins de Souza SIQUEIRA ${ }^{4}$ \\ Walene de Cássia SOUZA ${ }^{5}$ \\ Caroline Aparecida de Souza PEREIRA ${ }^{6}$ \\ Eric Batista FERREIRA ${ }^{2}$
}

1.MédicoIntensivista da Fundação Hospitalar do Município de Varginha-MG .

2. Professores da Universidade Federal de Alfenas-MG

3. Enfermeiro da Unidade Básica de Saúde Dr. José Marcos Xavier de Varginha-MG.

4.Aluna de Medicina da Faculdade Médica de Caratinga-MG.

5. Enfermeira da Fundação Hospitalar do Município de Varginha-MG.

6. Enfermeira da Casa de Saúde de Santa Fé, Três Corações-MG.

*Autor Correspondente: walne23@yahoo.com.br

Universidade Federal de Alfenas- UNIFAL-MG.

Recebido em: 15/08/2012 - Aprovado em: 15/12/2012 - Disponibilizado em: 30/12/2012

RESUMO: Introdução: A infecção hospitalar (IH), que atinge principalmente os Centros de Tratamento Intensivo (CTIs) das unidades hospitalares, é considerada um problema de saúde pública pela Organização Mundial de Saúde. Diante disso, este estudo objetivou determinar a prevalência de infecção hospitalar em um Centro de tratamento Intensivo. Metodologia: Trata-se de um estudo epidemiológico descritivo, do tipo transversal e retrospectivo, realizado no CTI para pacientes adultos de um Hospital Público do Sul de Minas Gerais. Participaram do estudo 62 pacientes. Os dados foram coletados por meio de registro nos prontuários referentes ao período de janeiro a abril de 2009. Resultados: A prevalência de IH no CTI foi de 19,35\%. Com relação à patologia de base, foi elevada a porcentagem de internações por doenças cardiovasculares $(50 \%)$. Os sítios de maior prevalência de $\mathrm{IH}$ foram as pneumonias $(50 \%)$ e infecção do trato urinário (33,3\%). Nas culturas realizadas, predominaram Staphylococcus sp (45,5) e os microrganismos Acinectobacter sp, Klebsiella sp e Pseudomonas sp (27,3\%). Os antimicrobianos mais utilizados foram ceftriaxona $(58,3 \%)$ e ampicilina sulbactam, ciprofloxacin, metronidazol, clindamicina (25\%). Os fatores de risco fortemente correlacionados com a ocorrência de infecção hospitalar no CTI foram a gravidade da doença de base, os procedimentos invasivos e o tempo de permanência no CTI. Conclusões: Os resultados deste estudo, apesar de ter sido realizado por um curto período de tempo, contribuem para mostrar como está a prevalência de IH em hospitais públicos e para fortalecer a importância de um programa de controle de infecção hospitalar efetivo com um maior envolvimento dos profissionais de saúde.

PALAVRAS-CHAVE: Infecção hospitalar. Centros de Terapia Intensiva. Prevalência. Hospital. Microrganismos.

\section{Evaluation of nosocomial infections in critically ill patients in an Intensive Care Unit}

ABSTRACT: Introduction: Hospital infection (HI), which primarily affects the Centers for Intensive Care Unit (ICU) of hospitals, is considered a public health problem by the World Health Organization. Thus, this study determined the prevalence of infection in a hospital Intensive Care Unit. Methodology: This is a descriptive epidemiological study, a cross-sectional and retrospective one in the ICU for adult patients of a public hospital in southern Minas Gerais state, Brazil. The study included 62 patients. Data were collected through the registration records from January to April 2009. 
Results: The prevalence of $\mathrm{HI}$ in the ICU was $19.35 \%$. Regarding the underlying pathology it was found a high percentage of admissions due to cardiovascular disease (50\%). The greatest prevalence of HI was pneumonia (50\%) and urinary tract infection (33.3\%). In cultures performed, Staphylococcus sp predominated (45.5\%), followed by Acinectobacter sp, Klebsiella sp and Pseudomonas sp (27.3\%). The most commonly used antimicrobial agents were ceftriaxone $(58.3 \%)$ followed by sulbactam ampicillin, ciprofloxacin, metronidazole, clindamycin (25\%). Risk factors strongly correlated with the occurrence of hospital infection in the ICU were the severity of underlying disease, invasive procedures and length of stay in ICU. Conclusions: The results of this study, despite having been held for a short period of time, help to show how is the prevalence of $\mathrm{HI}$ in public hospitals and to strengthen the importance of a program of hospital infection control effective with a greater involvement of health professionals.

KEYWORDS: Hospital Infection. Intensive Care Unit. Prevalence. Hospital. Microorganisms.

\section{INTRODUÇÃO}

A infecção hospitalar $(\mathrm{IH})$ representa

uma condição grave que influencia na morbimortalidade dos pacientes que permanecem internados. Em Centros de Terapia Intensiva (CTIs), os pacientes encontram-se acamados por tempo prolongado, imunodeprimidos, com doenças graves que necessitam de monitoramento invasivo e de uso de antibióticos de largo espectro, tornando-os mais suscetíveis às $\mathrm{IH}$ (DAVID, 1998).

Os pacientes internados nos CTIs abrangem um pequeno subgrupo dos pacientes hospitalizados, representando apenas de 5 a $10 \%$ do total, mas contribuem com mais de $20 \%$ dos episódios de infecção hospitalar (LIMA et al., 2007).

O risco de infecção é diretamente proporcional à gravidade da doença, condições nutricionais, à natureza dos procedimentos diagnósticos e/ou terapêuticos, bem como, ao tempo de internação, dentre outros aspectos (COUTO; PEDROSA; NOGUEIRA, 2003). Assim, nos CTIs, esses pacientes estão expostos a uma ampla variedade de microrganismos patogênicos, com doenças graves que necessitam de monitoramento invasivo e de uso de antimicrobianos de largo espectro, tornando-os mais suscetíveis às IH (COUTO; PEDROSA; NOGUEIRA, 2003).

As infecções hospitalares são apontadas como um dos mais importantes riscos aos pacientes hospitalizados. Isso justifica a inclusão dos índices de infecção hospitalar como um parâmetro de controle de qualidade do serviço prestado por um hospital (ANDRADE; ANGERAMI, 1999).

Diante dessa problemática, dos aspectos abordados e da vulnerabilidade dos pacientes internados no CTI, considerado área crítica, onde há possibilidade de maior número de infecção (GUIMARÃES; ROCCO, 2006), este estudo objetivou determinar a prevalência de infecção hospitalar, no Centro de Tratamento Intensivo, verificar a distribuição por topografia e por microrganismos e suas sensibilidades microbianas.

\section{MATERIAL E MÉTODOS}

Trata-se de um estudo descritivo, do tipo transversal e retrospectivo, realizado no CTI geral de uma Fundação Hospitalar do Sul de Minas Gerais. Foram incluídos no estudo todos os pacientes maiores de 18 anos admitidos no 
CTI Geral, no período de 5 de janeiro a 30 de abril de 2009. Foram excluídos os participantes que permaneceram na unidade por um período inferior a três dias. A população do estudo constituiu-se por 62 pacientes.

O Hospital onde foi realizado o estudo é público, dispõe de 127 leitos, sendo 10 destinados aos CTIs. É um centro de referência para atendimento de oncologia. O hospital mantém uma Comissão de Controle de Infecção hospitalar, com um programa de vigilância, de controle e de prevenção das infecções hospitalares.

Os dados foram coletados por meio da verificação de documentos referentes aos indicadores de $\mathrm{IH}$, das culturas realizadas no período do estudo, constantes do banco de dados da Comissão de Controle de Infecção Hospitalar do hospital. O instrumento de coleta de dados foi um formulário estruturado com a indicação do serviço, do número da cultura, da topografia da IH, dos tipos de microrganismos e da sensibilidade aos antimicrobianos.

Para o preenchimento do formulário da CCIH, a enfermeira integrante dessa comissão realizou a coleta dos dados por meio dos registros nos prontuários dos pacientes (evolução médica, de enfermagem e os resultados de exames laboratoriais e radiológicos).

A indicação de realização de cultura e antibiograma seguiu o protocolo hospitalar, mesmo na ausência de infecção hospitalar. Foram considerados espécimes de material para cultura: sangue (hemocultura), amostra brônquica, lesão cutânea, ponta de cateter venoso central, secreção uretral, secreção de feridas cirúrgicas, líquido ascítico e urina. O método de coleta de material para cultura seguiu as recomendações contidas na rotina elaborada pelo Laboratório de Microbiologia e pela CCIH da instituição.

Os dados coletados foram tabulados pelo software Excell®. Após, foi utilizado o software SPSS, Versão 9.0. $\mathrm{Na}$ análise estatística, foram utilizadas medidas simples como distribuição de frequências e percentuais. $O$ teste de qui-quadrado foi usado para comparar proporções.

$\mathrm{O}$ projeto de pesquisa segue os preceitos da resolução 196/96 do Conselho Nacional de Saúde e normas complementares e foram previamente aprovados pelo Comitê da Fundação Hospitalar do Município de Varginha-MG.

\section{RESULTADOS}

Durante o período do estudo, foram admitidos 62 pacientes no CTI do hospital, sendo que a taxa de incidência de infecção hospitalar foi de 19,35\% (12/62), com uma prevalência de 1,4 infecções por paciente. A proporção de infecção hospitalar encontrada $(19,35 \%)$ foi estatisticamente igual à referência 
nacional de $(15,5 \%)$, com $p=0,521$, de acordo com o Teste de qui-quadrado para proporções.

A taxa de ocupação dos leitos foi $95 \%$ e o tempo médio de permanência foi de 24 dias (com variações de 9 a 39 dias). Observamos que as IHs ocorreram em $66,7 \%$ no sexo masculino e a idade variou de 21 a 92 anos, sendo que $50 \%$ dos pacientes estavam na faixa etária de 36 a 48 anos.

Em relação aos óbitos e à alta dos pacientes internados com infecção hospitalar no CTI, $8(66,7 \%)$ receberam alta; $3(25 \%)$ foram a óbito; e 1 extremamente grave $(8,3 \%)$ foi transferido para outro hospital.
Os fatores preditivos para a ocorrência de infecção hospitalar foram os procedimentos invasivos como o cateterismo venoso central, o cateterismo urinário de permanência, a intubação orotraqueal, a traqueostomia, a utilização de ventilação mecânica e também do tempo de permanência no hospital.

As patologias de base responsáveis pela internação no CTI encontram-se na Figura 1.

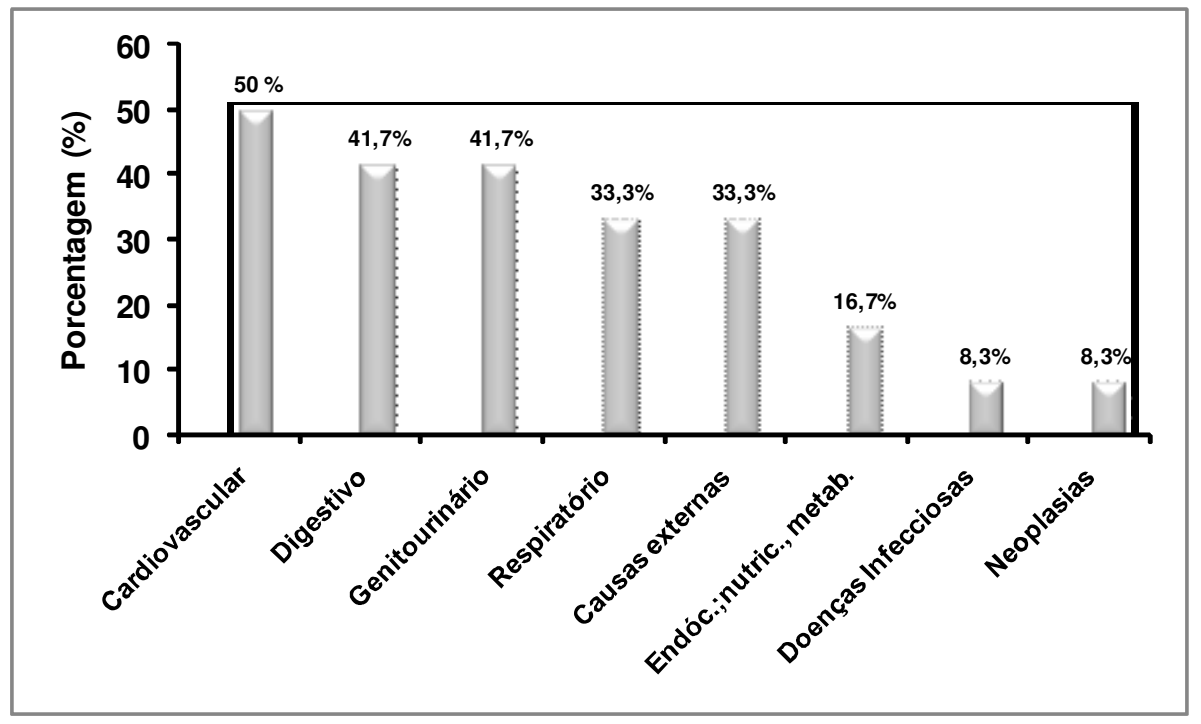

Figura 1 - Distribuição dos pa cientes a cometidos por infecção hospitalar segundo a patologia de base motiva dora da internação no CT Iem 2009.

Quanto à distribuição das infecções hospitalares por topografia, o principal sítio específico do processo infeccioso foi à pulmonar. O segundo lugar foi ocupado pela infecção do trato urinário como apresentado na figura 2. 


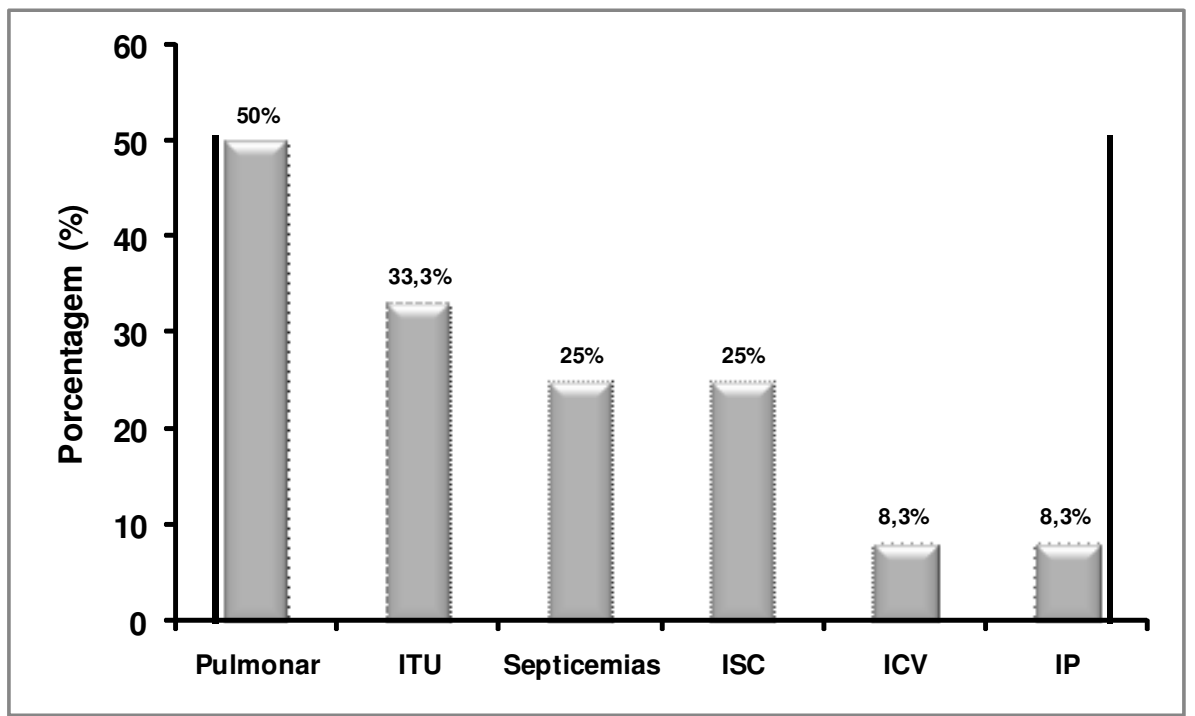

Figura 2 - Prevalência das infecções hospita lares segundo o sítio específico do processo infeccioso no CTI. ITU: Infecção do trato urinário; ISC:Infecção do sítio cirúrgico;ICV: Infecção cardiovascular; IP: Infecção peritoneal.

Em relação aos procedimentos paciente. Os dados estão apresentados na figura invasivos, verificamos que houve $23 \quad 3$.

procedimentos, que variaram de 1 a 3 por

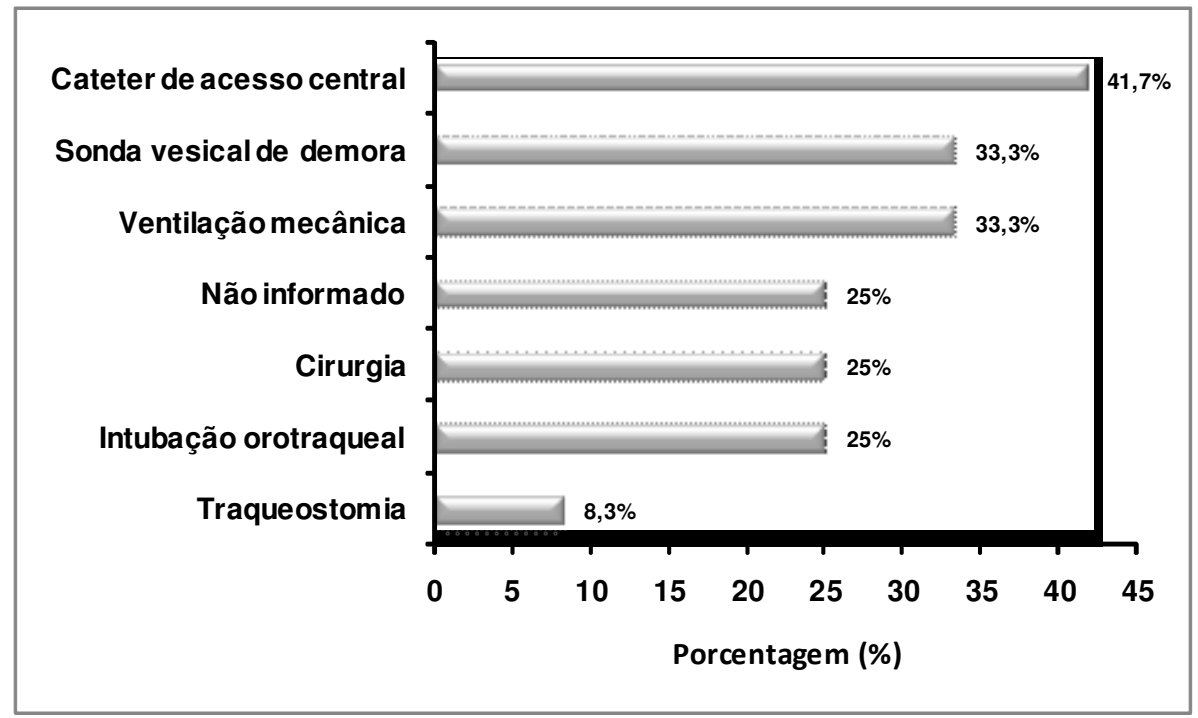

Figura 3- Distribuição dos procedimentos inva sivos por pacientes portadores de IH do CTI.

Foram realizadas 31 culturas com de 2 microrganismos por paciente. Os isolamento de 11 tipos de microrganismos principais microrganismos causadores de responsáveis pela $\mathrm{IH}$, sendo isolado uma média infecção foram o Staphylococcus sp $(5=45,5 \%)$ 
seguidos pelo Acinetobacter sp, Pseudomonas

sp e Klebsiella sp $(3=27,3 \%)$. Os microrganismos que apresentaram menor prevalência nos resultados das culturas foram:

Proteus sp, Streptococcus sp, Morganella morganii, Escherichia coli, Enterobacter sp, Staphylococcus aureus e Pseudomonas aeruginosa, todos representando $9,1 \% \quad(\mathrm{n}=1)$ dos casos.

Para todos os microrganismos isolados foi realizado o antibiograma. A tabela 1 apresenta a sensibilidade dos 4 microrganismos que prevaleceram no estudo dos antimicrobianos utilizados no CTI.

Tabela 1- Sensibilidade dos microrganismos de acordo com o antibiograma realizado para os pacientes com IH no CTI

\begin{tabular}{|c|c|c|c|c|}
\hline Antimicrobiano & Staphylococcus sp. & Pseudomonas sp & Klebsiella sp & Acinetobacter sp \\
\hline Amicacina & ++ & + & +++ & - \\
\hline Amoxicilina/clavulanato & +++ & & ++ & - \\
\hline Ampicilina & - & & - & - \\
\hline Ampicilina/sulbactam & +++ & & + & - \\
\hline Aztreonan & - & + & ++ & - \\
\hline Cefalotina & +++ & & + & - \\
\hline Cefepime & +++ & + & ++ & - \\
\hline Ceftazidima & +++ & +++ & ++ & - \\
\hline Ceftriaxona & - & - & + & - \\
\hline Ciprofloxacin & - & + & ++ & - \\
\hline Clindamicina & ++ & - & ++ & \\
\hline Gentamicina & + & + & ++ & - \\
\hline Imepenem & ++ & + & +++ & - \\
\hline Levofloxacin & ++ & - & +++ & ++ \\
\hline Linezolid & +++ & & & \\
\hline Meropenen & ++ & ++ & +++ & - \\
\hline Oxacilina & + & & & \\
\hline Piperacilina/tazobactam & +++ & + & & - \\
\hline Teicoplanina & ++ & & & \\
\hline Vancomicina & +++ & & & \\
\hline
\end{tabular}

A Tabela 2 apresenta os percentuais de Pseudomonas sp, Klebsiella sp e sensibilidade do Staphylococcus sp, Acinetobacter sp. 
Tabela 2. Percentual de suscetibilidade aos antimicrobianos das cepas isoladas de pacientes com IH no CTI.

\begin{tabular}{cccc}
\hline Antimicrobiano & $\mathbf{n}^{\mathbf{0}}$ cultura & Atividade & \% \\
\hline Vancomicina & 5 & 5 & 100 \\
Linezolid & 4 & 3 & 75 \\
Ceftazidima & 9 & 5 & 56 \\
Amoxicilina/Clavulanato & 4 & 2 & 50 \\
Levofloxacin & 8 & 4 & 50 \\
Teicoplanina & 2 & 1 & 50 \\
Amicacina & 9 & 4 & 44 \\
Cefepime & 9 & 4 & 44 \\
Clindamicina & 5 & 2 & 40 \\
Imepenem & 10 & 4 & 40 \\
Meropenen & 10 & 4 & 40 \\
Ampicilina/Sulbactam & 8 & 3 & 38 \\
Cefalotina & 6 & 2 & 33 \\
Gentamicina & 15 & 5 & 33 \\
Piperacilina/Tazobactam & 7 & 2 & 29 \\
Aztreonan & 8 & 2 & 25 \\
Oxacilina & 4 & 1 & 25 \\
Ciprofloxacin & 12 & 2 & 17 \\
Ceftriaxona & 9 & 1 & 11 \\
Ampicilina & 3 & 0 & 0 \\
\hline
\end{tabular}

Em relação ao número de variou de 1 a 4 . Os antimicrobianos mais antimicrobianos utilizados pelos pacientes, utilizados encontram-se na figura 4. houve uma média de 2,8, sendo que o número

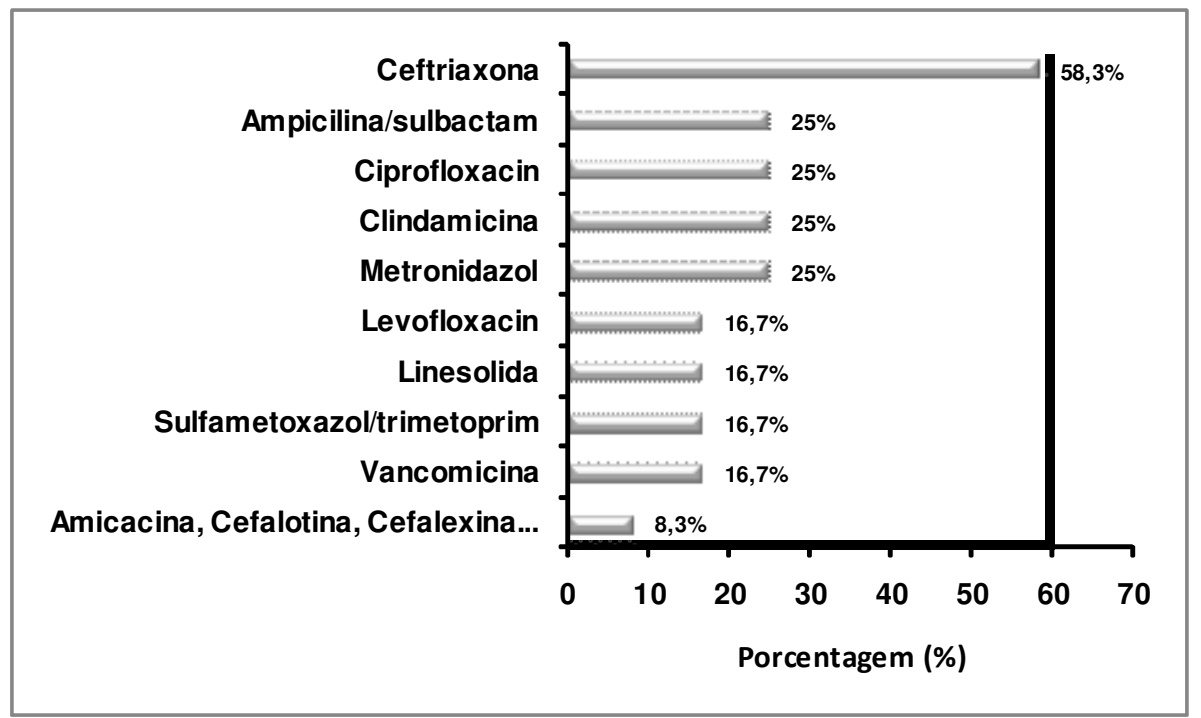

Figura 4- Utilização de antimicrobia nos pelos pacientes com infecção hospita lar no CTI. 


\section{DISCUSSÃO}

No estudo realizado, verificamos que houve uma taxa de IH de 19,35\%, com a ocorrência de 1,4 episódios. De acordo com estudos realizados no Brasil, o índice de infecção conforme dados está em torno de $15,5 \%$, o que corresponde a 1,18 episódios de infecção por paciente internado com IH nos hospitais brasileiros. Esses índices têm se mantido altos, mesmo com a legislação vigente no país. Além disso, considera-se mais um agravante o fato das instituições de saúde pública possuírem a maior taxa de IH no país, em torno de 18,4\% (MOURA et al., 2007).

Mas as taxas de IH em CTI variam entre 18 a $54 \%$, sendo cerca de cinco a dez vezes maior do que em outras unidades de internação de um hospital. É responsável por 5 a $35 \%$ de todas as IHs e por, aproximadamente, $90 \%$ de todos os surtos que ocorrem nessas unidades (GUSMÃO et al., 2004)

Nos EUA, estima-se que de 5\% até $35 \%$ dos pacientes admitidos numa UTI podem ser acometidos por infecção hospitalar (VICENT et al. 1995). No International Study of the Prevalence and Outcomes of Infection in Intensive Care Units (EPIC II), realizado por meio de um estudo de prevalência de infecção em CTI nos países da Europa, os pesquisadores verificaram que a prevalência de $\mathrm{IH}$ foi de $51 \%$ e $71 \%$ dos pacientes que estavam recebendo antimicrobiano

(VINCENT et al., 2009). No Brasil, por exemplo, em 1994, em um estudo multicêntrico do Ministério da Saúde, realizado em 99 hospitais terciários, localizados nas capitais, a taxa de infecção, nas UTIs de adulto, foi em média de 38,9\% (OLIVEIRA et al., 2009).

Segundo o International Study of the Prevalence and Outcomes of Outcomes of Infection in Intensive Care Units (VICENT et al., 2009), realizado com 13796 UTIs em 75 países, na América do Sul e Central, houve a participação de 1290 pacientes, sendo que o índice de infecção foi de 60,3 , o mais alto índice encontrado no estudo.

No Brasil, apesar da criação da primeira Comissão de Controle de Infecção hospitalar ligada ao Ministério da Saúde há aproximadamente quase 30 anos, o país não tem dados sobre quantas pessoas morrem anualmente em decorrência dessas infecções ou qual seria o índice de infecção, por exemplo, aceitável na UTI, no berçário ou para doentes que estejam com pneumonia. No entanto, informações retiradas de estudos realizados por todo o país pela Associação Nacional de Biossegurança (Anbio) trazem números alarmantes - em média, $80 \%$ dos hospitais não fazem o controle adequado. $\mathrm{O}$ índice de $\mathrm{IH}$ varia de $14 \%$ e $19 \%$, podendo chegar, dependendo da unidade, a $88,3 \%$; e cem mil 
pessoas morrem em média por ano em decorrência das infecções (NOBLAT, 2011).

As diferenças das taxas de ocorrência de IH em CTI são devido ao método de vigilância utilizado (OLIVEIRA et al., 2009) de acordo com a população atendida; em razão da gravidade da patologia de base; do uso frequente de sedação; das alterações no nível de consciência; e dos múltiplos procedimentos invasivos, principalmente das vias respiratórias (MARTINS, 2006).

Assim, as patologias de base motivadoras da internação em CTI são importantes nos riscos de infecção hospitalar. Portanto, em nosso estudo, as patologias de base que levaram os pacientes à internação no CTI variaram de uma a cinco e as mais prevalentes foram devido às doenças cardiovasculares, as pulmonares, as do aparelho digestivo e geniturinário. Alguns pacientes apresentavam processos infecciosos prévios à admissão no CTI, originados em outros serviços ou unidades hospitalares ou decorrentes de procedimentos invasivos e da debilidade do organismo. Essas situações requerem o uso de antimicrobianos, o que é um fator de risco comprovado para a seleção de resistência de microrganismos, tendo em vista que os pacientes colonizados ou infectados por microrganismos, frequentemente têm uma história de hospitalização prolongada ou antibioticoterapia prévia na comunidade ou hospital (MARTINS, 2001).

Com relação aos dados referentes aos sítios específicos do processo infeccioso, verificamos que a infecção de maior prevalência foi a pulmonar (50\%). Comparando esse resultado com as patologias de base, que levaram os pacientes à internação no CTI, houve uma correlação com as doenças do trato respiratório. Portanto, o uso de ventilação mecânica, associado ao tempo prolongado de utilização desse procedimento e à debilidade do organismo dos pacientes, pode ter colaborado para as ocorrências de IHs.

No estudo realizado por Padrão et al. (2010), em Campos de Goitacazes-RJ, o trato respiratório também foi o principal fator responsável pela ocorrência de infecção hospitalar na UTI. Amarante (1997) aponta que a utilização de ventilação mecânica, de cânulas orotraqueais e de traqueostomias está diretamente associada à ocorrência de pneumonias. Esses pacientes, segundo o autor, podem apresentar taxas de $\mathrm{IH}$ entre 7 a 21 vezes maiores que os pacientes sem dispositivos de assistência ventilatória. Assim, em nosso estudo, foram isolados 10 microrganismos da secreção traqueal que foram responsáveis pela infecção pulmonar. Os microrganismos isolados foram o Acinetobacter sp, Pseudomonas sp e Staphylococcus sp (n=2; 20\%); Pseudomonas 
aeruginosa, Enterobacter sp, Proteus sp e Staphylococcus aureus $(\mathrm{n}=1 ; 10 \%)$.

Segundo dados da Agencia Nacional de Vigilância Sanitária (ANVISA, 2000), 25\% a $40 \%$ dos pacientes sob ventilação mecânica por períodos superiores a 48 horas desenvolvem pneumonia. Portanto, nossos resultados foram compatíveis com o descrito pela literatura.

Os sítios de maior prevalência das IHs encontrados no CTI do hospital estudado foram semelhantes também aos apresentados em um estudo multicêntrico realizado em hospitais gerais nas capitais brasileiras e no Distrito Federal, por Prade et al.(1995). Esses autores detectaram como principais sítios de infecções o trato respiratório, incluindo pneumonias, o trato urinário e as septicemias.

O segundo tipo de infecção prevalente em nosso estudo foi a do trato urinário, correspondendo a 33,3\% dos pacientes. Foram isolados 4 microrganismos do trato urinário responsáveis pela $\mathrm{IH}$. Os microrganismos foram o Acinetobacter sp, a Escherichia coli, o Streptococcus sp e a Klebsiella sp, sendo cada um correspondendo a $25 \%(n=1)$.

Segundo vários estudos, as infecções do trato urinário (ITU) correspondem a cerca de $70 \%$ a $88 \%$ dos casos de infecção hospitalar em pacientes submetidos ao cateterismo vesical. A duração do cateterismo é um dos fatores que contribuem para a ocorrência da infecção urinária. Mas a infecção depende das características do microrganismo causador e da defesa do hospedeiro (MOURA et al., 2007).

Outro tipo de infecção prevalente foi associado ao uso de cateter de acesso venoso central $(41,7 \%)$. Foram isolados da ponta do cateter 4 microrganismos como o Staphylococcus sp e Klebsiella $\mathrm{sp}(\mathrm{n}=2 ; 50 \%)$. Esses agentes foram responsáveis pela infecção da corrente sanguínea (25\%).

Com relação aos pacientes submetidos à cirurgia (25\%), de um foi isolado da ferida cirúrgica a Pseudomonas sp, $(\mathrm{n}=1 ; 33,3 \%)$; de outro, a Morganella morganii $(\mathrm{n}=1 ; 33,3 \%)$ e, de outro, o Staphylococcus sp $(\mathrm{n}=1 ; 33,3 \%)$.

$$
\text { Portanto, dos } 11 \text { tipos de }
$$
microrganismos isolados responsáveis por $\mathrm{IH}$, verificamos uma predominância de 45,5\% de Staphylococcus sp; 27,3\% do Acinetobacter sp, da Pseudomonas sp e da Klebsiella sp; e $9,1 \%$ para os outros microrganismos.

Segundo Gabliarbi et al. (2000), a ocorrência de infecção depende das características do microrganismo causador, do tamanho do seu inóculo e da defesa do hospedeiro. Esses autores verificaram que os microrganismos Pseudomonas aeruginosa, Klebsiella sp, Proteus sp e o Enterobacter sp estão entre os principais agentes causadores de infecção hospitalar. Portanto, mostrando resultados semelhantes aos encontrados no presente estudo.

Estudos epidemiológicos, realizados por Wang e Chang (2002) e Menezes et al. 
(2007), também mostram que os microrganismos gram-negativos mais comumente isolados em Unidades de Terapia Intensiva (UTI) foram Pseudomonas aeruginosa, Escherichia coli, Klebsiella sp e Acinetobacter sp. Os dados coincidem com os encontrados em nosso estudo, confirmando a importância mundial desses patógenos.

Além dos microrganismos gramnegativos, verificamos uma elevada porcentagem de microrganismos grampositivos, sendo predominantes as cepas de Staphylococcus sp $(45,5 \%)$ e uma cepa de Staphylococcus aureus. O Staphylococcus é considerado um patógeno humano oportunista e com frequência está associado às infecções hospitalares. De acordo com o National Nosocomial Infections Surveillande do Center for Disease Control and Prenvention (CDC), desde 1999, a proporção de $S$. aureus resistente à meticilina/oxacilina (Staphylococcus aureus meticilina-resistentes MRSA) ultrapassa $50 \%$ entre os pacientes internados em UTI. No Brasil, as infecções hospitalares causadas por $S$. aureus resistentes à meticilina/oxacilina também são elevadas, correspondendo de $40 \%$ a $80 \%$, principalmente nas UTIs (MOURA et al, 2007).

Com relação à sensibilidade e resistência dos microrganismos, constatamos que, dentre todos os antimicrobianos utilizados no CTI, os que apresentaram menor índice de resistência microbiana foram a vancomicina e a linezolida. Estudos anteriores evidenciaram a predominância da sensibilidade das cepas de Staphylococcus aureus, isoladas de pacientes da UTI à vancomicina (100\%) e uma baixa sensibilidade à oxacilina (MOURA et al. 2007). Esses resultados também estão de acordo com nossos achados, onde encontramos uma sensibilidade de $100 \%$ à vancomicina e de $25 \%$, à oxacilina. Essa resistência à oxacilina varia conforme a região ou mesmo de acordo com o hospital analisado, devendo ser avaliada localmente (AMYES; GEMELL, 1997).

A vancomicina é um antibiótico glicopeptídeo, muito utilizado para várias infecções por bactérias gram-positivas em pacientes alergicos à penicilina ou resistêntes aos $\beta$-lactâmicos em ambiente hospitalar. É um antimicrobiano de reserva terapêutica, sendo utilizado quando o paciente não responde aos antimicrobianos padrões. Entretanto a crescente resistência dos microrganismos aos antimicrobianos pode levar ao indesejado cenário de tempos atrás, quando infecções bacterianas eram em sua maioria, fatais. Em 2002, foi detectado o primeiro caso de resistência à vancomicina e, em 2004, 4 casos de resistência microbiana foram relatados nos Estados Unidos (SCHITO, 2006). No Brasil, alguns estudos prospectivos em UTIs mostram taxas entre 14 e $25 \%$ de colonização retal, em geral em pacientes com uso prévio de antibióticos (vancomicina) e 
com história de longa permanência hospitalar (ANVISA,2007). Portanto, uma vez que encontramos uma boa susceptibilidade dos microrganismos a essa classe de antibióticos, os profissionais de saúde devem utilizá-lo de forma racional, procurando evitar a resistência.

A linezolida é uma nova classe de antimicrobiano, oxazolidinona, utilizado para tratamento de infecções por bactérias grampositiva, incluindo os Staphylococcus aureus resistentes à meticilina/oxacilina e Enterococcus resistentes a vancomicina (TSIODRA et al., 2001). Nossos resultados mostraram que os microrganismos apresentaram sensibilidade aproximadamente semelhante a esses antimicrobianos, quando comparados com a vancomicina.

Ainda em relação ao perfil de utilização dos antibacterianos, nossos resultados mostram que a especialidade farmacêutica antibacteriana mais prescrita no CTI, no período de estudo, foi a ceftriaxona $(58,3 \%)$, uma cefalosporina de $3^{\mathrm{a}}$ geração. Estudos realizados em UTI de Brasília, por Carneiro (2006) e, em Fortaleza, por Sousa et al. (2011), mostram resultados semelhantes, sendo a ceftriaxona o fármaco prevalente $(12,9 \%$ e $31,9 \%$ respectivamente).

Apesar de a ceftriaxona ser o antimicrobiano mais utilizado, nossos resultados mostram uma sensibilidade à ceftriaxona muito pequena ( $11 \%)$. Nos últimos anos, com o avanço da tecnologia, houve grande mudança na antibioticoterapia ao surgirem agentes antimicrobianos mais potentes e eficazes. As cefalosporinas introduzidas no mercado, além de terem um amplo espectro antibacteriano, trouxeram ganhos em termos de posologia, farmacocinética e outras propriedades, como as relacionadas com atividades imunomoduladoras (SOUSA et al., 2011).

Entretanto, contrabalanceando esses ganhos, existe o perigo iminente pelo uso inadequado desses novos fármacos, pois aceleram a emergência de infecções por bactérias multirresistentes que antes poderiam ser facilmente debeladas.

O uso irracional de antimicrobianos é relatado em várias partes do mundo, sendo um problema ainda bastante atual. A utilização inadequada de antimicrobianos também assume importante papel no crescimento alarmante, em todo o mundo, da resistência bacteriana aos antibióticos. Segundo dados da Organização Pan-Americana de Saúde (OPAS), em determinadas áreas da América Latina, cerca de $75 \%$ dos antibióticos são prescritos inadequadamente e a resistência bacteriana tem sido constatada em até $50 \%$ das cepas isoladas (RABELO, 2002).

Em nosso estudo, verificamos que o microrganismo que apresentou maior resistência foi o Acinetobacter sp com apenas 3\% de sensibilidade aos antimicrobianos. Esses dados estão de acordo com trabalhos anteriores 
(PONTES et al., 2006; JONES et al., 2004) que mostram uma alta resistência destes microrganismos à maioria dos antimicrobianos. De acordo com Levin (2008), o tratamento de infecções por Acinetobacter $\mathrm{sp}$ é geralmente realizado com imipenem embora não haja ensaios clínicos randomizados que embasem essa recomendação. No caso de resistência a essa classe, situação cada vez mais frequente, as opções mais estudadas são as polimixinas e ampicilina/sulbactam. Neste estudo, verificamos uma resistência do Acinetobacter sp não só aos carbapenêmicos (imipenem e meropenem) como também à ampicilina/sulbactam. Esse microrganismo apresentou uma sensibilidade parcial à levofloxacina, uma fluoroquinolona de terceira geração. Em 2000, Joly-Guillou e colaboradores avaliaram a ação da levofloxacina contra o Acinetobacter, já preocupados com a multirresistência. Jones et al. (2004) mostraram uma média de $50 \%$ de sensibilidade do Acinetobacter sp às fluoroquinolonas em Unidade de Terapia Intensiva, entre os países dos Estados Unidos, Canadá, Itália e Alemanha, no período de 20002002.

Assim, as infecções hospitalares são consideradas agravos que acometem o paciente após admissão em um hospital, com tratamento de alto custo. Essas infecções são consideradas como importantes fatores de complicação no tratamento de pacientes internados uma vez que podem causar mais sofrimento para o paciente e para a sua família, contribuir para o aumento das taxas de morbidade, de mortalidade e de tempo de permanência no hospital, como também elevar custos no tratamento pósinfecção. Considerando a magnitude do problema das infecções em CTI no Brasil, torna-se imprescindível a manutenção de uma vigilância permanente por parte das Comissões de Controle de Infecção Hospitalar do hospital, conforme preconizado pelo Programa Nacional de Controle de Infecção Hospitalar do Ministério da Saúde.

Portanto, a vigilância epidemiológica, o estabelecimento de protocolos clínicos, a utilização de medidas de isolamento, de materiais e equipamentos adequados, a higienização do ambiente, a identificação de bactérias multirresistentes, a antibioticoterapia adequada, o treinamento da equipe multiprofissional, a implementação de medidas de controle, são fatores importantes e determinantes que podem impactar sobre os resultados, com redução das taxas de prevalência de infecção hospitalar.

\section{CONCLUSÕES}

A taxa de infecção hospitalar foi de 19,35\% em 62 pacientes. O sítio de infecção mais comum foi o trato respiratório, seguido das infecções do trato urinário. O tempo médio de internação foi de 24 dias. Nas culturas realizadas, predominaram Staphylococcus sp, 
Pseudomonas sp, Acinetobacter sp e Klebsiella

$s p$.

Os microrganismos sensíveis aos antimicrobianos mais comuns, responsáveis por infecções, foram o Staphylococcus sp e Klebsiella sp. O identificado como microrganismo resistente mais frequente causador de IH foi o Acinetobacter sp. O antimicrobiano mais utilizado foi a ceftriaxona, mas com uma sensibilidade de apenas $11 \%$.

Os resultados deste estudo, apesar de ter sido realizado por um período de tempo curto, contribuem para mostrar como está a prevalência de IH em hospitais públicos, fortalecendo a importância de um programa de controle de infecção hospitalar efetivo com um maior envolvimento dos profissionais de saúde.

\section{REFERÊNCIAS}

1.AMARANTE, J.M.B. Infecções do trato respiratório. In: Rodrigues, E.A.O.C. et al. Infecções hospitalares: prevenção e controle. São Paulo: Sarvier; 1997. p. 168-88.

2.AMYES, S.G.B.; GEMELL, C.G. Antibiotic resistance. J. Med.Microbiol., v. 46, p. 43670, 1997.

3.ANDRADE, D.A.; ANGERAMI, E. L. S. Reflexões acerca das infecções hospitalares às portas do terceiro milênio. Medicina, Ribeirão Preto, v. 32, p. 492-497, 1999.

4.ANVISA - Agência Nacional de Vigilância Sanitária. Curso básico de controle de infecção hospitalar: Caderno B: Principais síndromes infecciosas e hospitalares. Brasília: ANVISA; 2000.

5.ANVISA - Agência Nacional de Vigilância Sanitária. Surto de Enterococo Resistente à vancomicina em Estabelecimentos de Assistência à Saúde Brasília: ANVISA; 2007. http://www.anvisa.gov.br/servicosaude/control e/reniss/manual\%20_controle_bacterias.pdf

6.CARNEIRO, J.C.O. Padrão de consumo de antibacterianos em uma UTI geral: correlação com a resistência bacteriana. 2006. Dissertação (Mestrado) - Faculdade de Medicina, UNB, Brasília, 2006.

7.COUTO, R.C.; PEDROSA, T.M.G.; NOGUEIRA, J.M. Infecção hospitalar epidemiologia, controle e tratamento. $3^{\mathrm{a}} \mathrm{Ed}$. Rio de Janeiro: Editora Médica e Científica; 2003.

8.DAVID, C.M.N. Infecção em UTI. Medicina. Ribeirão Preto, v. 31, p.337-48, 1998.

9.GABLIARBI , E.M.D.B.; FERNANDES, A.T.; CAVALCANTE, N.J.F. Infecção do trato urinário. In: FERNANDES, M.O.V.; RIBEIRO FILHO, N. Infecção hospitalar e suas interfaces na área da saúde. $1^{\mathrm{a}} \mathrm{Ed}$. São Paulo (SP): Atheneu; 2000. p. 459-78.

10.GUIMARÃES, M.M.Q.; ROCCO, J.R. Prevalência e prognóstico dos pacientes com pneumonia associadas a ventilação mecânica em um hospital universitário. Rev. Bras. Pneumol., v. 22, n.4, p.339-46, 2006.

11.GUSMÃO, M.E.N.; DOURADO, I.; FIACCONE, R.L.; SALVADOR, C. Nosocomial pneumonia in the intensive care unit of a brazilian university hospital: na analysis of the time span from admission to disease onset. Am. J. Infect. Control., v. 32, n.4, p.209-14, 2004.

12.JOLY-GUILLOU, M.L.; WOLFF, M.; FARINOTTI, R.; BRYSKIERD, A.; CARBON, C. In vivo activity of levofloxacin alone or in combination with imipenem or amikacin in a mouse model of Acinetobacter baumannii pneumonia. J. Antimicrob. Chemother., v. 46, n.5, p. 827-830, 2000.

13.JONES, M.E.; DRAGHI, D.C.; THORNSBERRY, C.; KARLOWSKY, J.A.; SAHM, D.F.; WENZEL, R.P. Emerging 
resistance among bacterial pathogens in the intensive care unit-a European and North American Surveillance study (2000-2002).

Ann. Clin. Microbiol. Antimicrob., n.3, v.14, p. 1-11, 2004.

14.LEVIN, A.S. Overcoming antimicrobial resistance. The Braz. J. Infect .Dis., v. 12, p. 10-21, 2008.

15.LIMA, M.E.; ANDRADE, D.; HASS, V.J. Avaliação prospectiva da ocorrência de infecção em pacientes críticos de unidades de terapia intensiva. Rev. Bras. Terap. Intens., v.19, n.3, p.342-7, 2007.

16.MARTINS, M.A. Conceitos gerais e terminologia básica em epidemiologia hospitalar e controle de infecção. In: MATINS, M.A. Manual de infecção hospitalar: epidemiologia, prevenção e controle. Belo Horizonte: Medsi; 2001. p. 16-26.

17.MARTINS， P. Epidemiologia das infecções em centro de terapia intensiva de adulto. 2006. 96f Tese (Doutorado em Medicina Tropical) - Escola de Medicina, UFMG, Belo Horizonte, 2006.

18.MENEZES, E. A.; SÁ, K. M.; CUNHA, F. A.; ÂNGELO, M. R. F.; OLIVEIRA, I. R.N.; SALVIANO M. N. C. Freqüência e percentual de suscetibilidade de bactérias isoladas em pacientes atendidos na Unidade de Terapia Intensiva do Hospital Geral de Fortaleza. J. Bras. Patol. Med. Lab., v. 43; n. 3, p. 149155, 2007.

19.MOURA, M.E.B.; CAMPELO, S.M.A.; BRITO, F.C.P.B. et al. Infecção hospitalar: estudo de prevalência em um hospital público de ensino. Rev. Bras.

Enferm., v.60, n.4, p.4116,-21, 2007.

20.NOBLAT, R. Infecção hospitalar no Brasil mata mais do que guerra do Vietnã. O Globo, Rio de Janeiro, 25 de set. 2011. Disponível em: http://oglobo.globo.com. Acessado em janeiro/2012. Jornal eletrônico.

21.OLIVEIRA, O.A.; OLIVEIRA, A.L.L.; PONTES, E.R.J.C.; OLIVEIRA, S.M.V.L.; CUNHAS, R.V. Epidemiologia da infecção hospitalar em unidade. Rev. Panam. Infectol., v.11, n.2, p.32-37, 2009.

22.PADRÃO, M.C.; MONTEIRO, M.L.; MACIEL, N.R.; VIANA, F.F.C.F. Prevalência de infecções hospitalares em unidade de terapia intensiva. Rev. Bras. Clin. Med., v.8, n.2, p.125-28, 2010.

23.PONTES, V. M. O.; MENEZES, E. A.; CUNHA, F. A.; ÂNGELO,M. R. F.;

SALVIANO, M.N.C.; OLIVEIRA, I. R. N.

Perfil de Resistência de Acinetobacter

baumannii a Antimicrobianos nas Unidades de

Terapia Intensiva e Semi-Intensiva do Hospital Geral de Fortaleza. RBAC, v. 38 n. 2, 123-26, 2006.

24.PRADE, S.S.; OLIVEIRA, S.T.; RODRIGUEZ, R.; NUNES, F.A.; NETTO, E.M.; FELIX, J.Q. et al. Estudo Brasileiro da Magnitude das infecções hospitalares em hospitais terciários. Rev. Control. Inf. Hosp., v.2, p.11-25, 1995.

25.RABELO, J.I.C.R. Normatização do uso racional de antimicrobianos. Secretaria de Saúde do Estado do Ceará. Fortaleza, 2002.

26.SOUSA, P.C.P.; ROCHA, M.V.A.P.; SOUSA, F.S.P.; NOGUEIRA, N.A.P.

Utilização de antibacterianos em Unidade de Terapia Intensiva. Inter Science Place, ano 4, n. 18, p.1-15, 2011.

27.SCHITO G. C. The importance of the development of antibiotic resistance in Staphylococcus aureus. Clin. Microbiol. Infect., v. 12 n.1 p.3-8, 2006.

28.TSIODRAS, S.; HOWARD, S.G.; SAKOULAS, G.; ELIOPOULOS, G.M.; WENNERSTEN, C.; VENKATARAMAN, L. ; MOELLERING, R.C.; FERRARO, M. J. Linezolid resistance in a clinical isolate of Staphylococcus aureus. The Lancet, v. 358, n. 9277, p. 207-08, 2001.

29.VINCENT, J.L.; BIHARI, D.J.; SUTER, P.M.; BRUINING, H.A.; WHITE, J.; NICOLAS-CHANOIN, M.H. et al. The prevalence of nosocomial infection in intensive care units in Europe. Results of the European 
prevalence of infection in intensive care (EPIC) study. JAMA, v. 274, p.639-44, 1995.

30.VICENT, J.L.; RELLO, J.; MARSHALL, J. et al. International study of the prevalence and outcomes of infection in Intensive Care Units. JAMA, v. 302, n.21, p. 2323-29, 2009.

31.WANG, H.; CHANG, M. Surveillance for antimicrobial resistance among clinical isolates of gram-negative bactéria from intensive care unit patients in China, 1996 to 2002. Diagn. Microbiol. Infect. Dis., v. 51, n. 1234-41, 2004. 